\title{
Neural Substrates of Response-Based Sequence Learning Using fMRI
}

\author{
Amanda Bischoff-Grethe ${ }^{1,2}$, Kelly M. Goedert ${ }^{3}$, Daniel T. Willingham ${ }^{4}$, \\ and Scott T. Grafton 5
}

\begin{abstract}
Representation of sequential structure can occur with respect to the order of perceptual events or the order in which actions are linked. Neural correlates of sequence retrieval associated with the order of motor responses were identified in a variant of the serial reaction time task in which training occurred with a spatially incompatible mapping between stimuli and finger responses. After transfer to a spatially compatible version of the task, performance enhancements indicative of learning were only present in subjects required to make finger movements in the same order used during training. In contrast, a second group of subjects performed the compatible task using an identical sequence of stimuli (and different order of finger
\end{abstract}

\section{INTRODUCTION}

Motor learning is the change in capacity to perform skilled movements that achieve behavioral goals in the environment. A fundamental and unresolved question in neuroscience is whether there are specific neural systems for representing sequential motor responses. Defining such systems with brain imaging and other methods requires a careful delineation of what specifically is being learned for a given sequencing task. As one learns to tap the fingers in a particular order, there can be enhanced performance due to a number of factors related to the level of skill representation. For example, in a sequence learning task such as the serial reaction time task (SRT), in which four fingers are used to press keys in response to visual cues that are presented in a repeating order, the subject might be learning where the next visual cue would occur in a sequence, irrespective of the particular motor command or outcome of a response. It is becoming clear that numerous experimental factors can determine the level of representation (perceptual or response based) of a sequence. It is known that over the course of learning improvements in performance can initially be based on perceptual

\footnotetext{
${ }^{1}$ San Diego VA Healthcare System, ${ }^{2}$ University of California, ${ }^{3}$ Pacific Lutheran University, ${ }^{4}$ University of Virginia, ${ }^{5}$ Dartmouth College
}

movements) as in training. They demonstrated no performance benefit, indicating that learning was response based. Analysis was restricted to subjects demonstrating low recall of the sequence structure to rule out effects of explicit awareness. The interaction of group (motor vs. perceptual transfer) with sequence retrieval (sequencing vs. rest) revealed significantly greater activation in the bilateral supplementary motor area, cingulate motor area, ventral premotor cortex, left caudate and inferior parietal lobule for subjects in the motor group (illustrating successful sequence retrieval at the response level). Retrieval of sequential responses occurs within mesial motor areas and related motor planning areas.

knowledge and progress to response-based knowledge (Bapi, Doya, \& Harner, 2000). Whether instructions are given as spatial or symbolic cues will also determine the degree to which subjects learn at a perceptual or response based level (Koch \& Hoffmann, 2000). Historically, it has been observed that a sequencing skill acquired in one hand can demonstrate complete transfer to the opposite hand, or between individual finger and whole arm movements, suggesting that learning might occur at either a perceptual level (Keele, Jennings, Jones, Caulton, \& Cohen, 1995; Cohen, Ivry, \& Keele, 1990) or with respect to a goal-relevant outcome. Recent behavioral experiments where training occurs with different types of stimuli show that subjects show no behavioral cost to switching stimulus modality provided that the same motor response is generated, arguing that sequence learning under some training conditions is not perceptually based (Hazeltine, 2002; Willingham, Wells, Farrell, \& Stemwedel, 2000; Nattkemper \& Prinz, 1997). Recent work by Hazeltine (2002) provides clear evidence that a sequence can be linked to the outcome of the movement rather than the movement itself. Together, these studies argue that depending on the specific training design, subjects are learning specific muscle patterns to generate movements, remembering specific stimuli to represent sequential behavior, learning an abstract motor goal (a sequence of responses in a relative order), or learning the environ- 
A
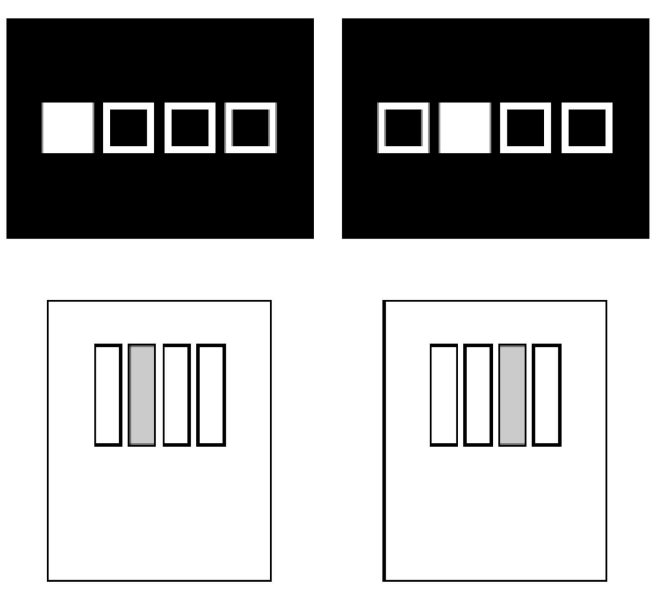

B
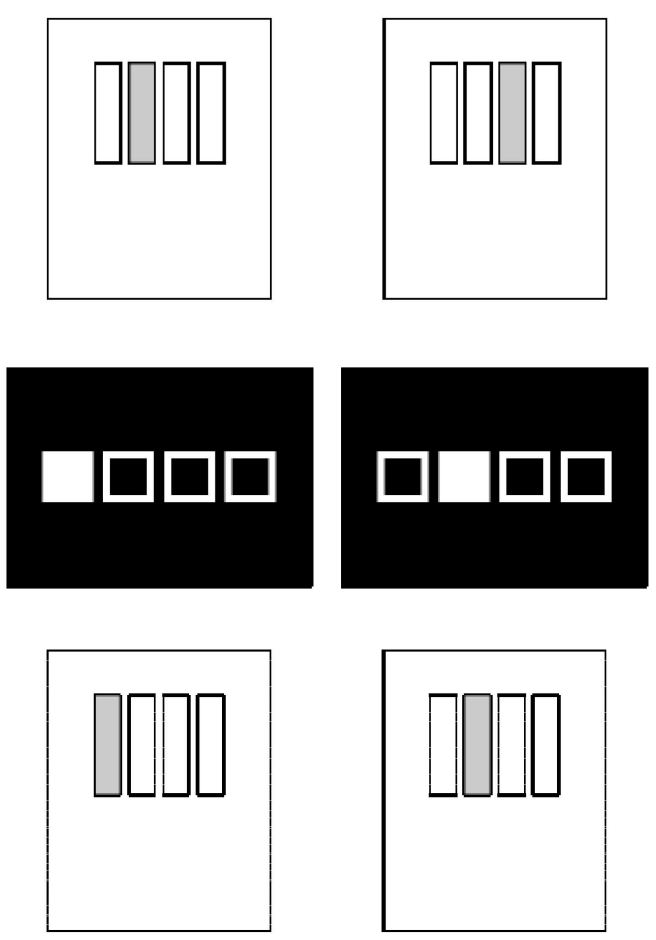
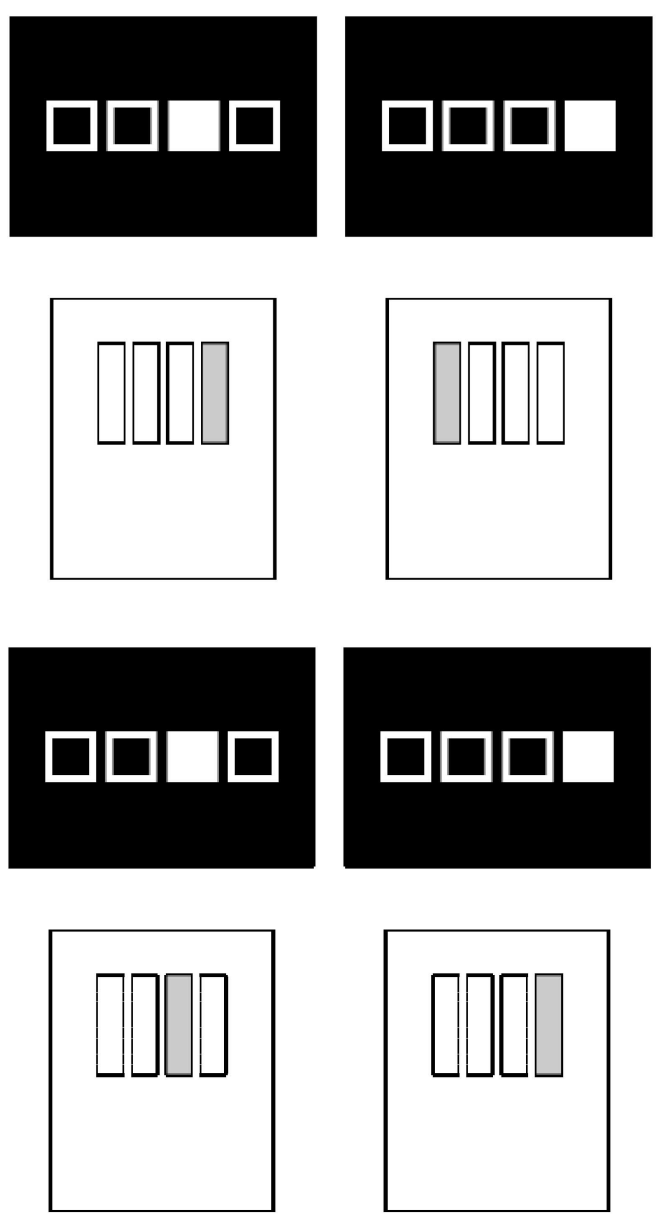

Figure 1. Diagram of screen display and button presses for (A) incompatible and (B) compatible sensorimotor mapping. In the incompatible mapping condition, subjects respond by pressing one key to the right of the illuminated stimulus. If the stimulus is on the far right, subjects respond with the index finger. In the compatible mapping condition, the response is directly correlated to the illuminated target. The thumb was not used in this task.

mental consequence of an action. These levels of sequence representation have not been considered in nearly all previous human neuroimaging studies of sequence learning. In most studies, it is assumed that learning is linked to sequential motor responses, and that methods such as the SRT task are directly linked to procedural motor learning. Given the multiple levels across which a sequence can be represented, the current imaging study was performed to firmly establish which functional brain changes with learning correspond specifically to response-based learning and to eliminate the possibility that the effects were due to perceptual learning.

To clearly demonstrate that subjects can learn to make sequential movements that are specific to the response rather than to perceptual stimuli, Willingham (1999) developed an incompatible version of the SRT task in which subjects were trained on a spatially incompatible mapping. In this mapping, the first spatial stimulus was a cue to respond with the second finger, the second stimulus was a cue to respond to the third finger, the third stimulus was a cue to respond with the fourth finger, and the fourth stimulus was a cue to respond with the first finger, as shown in Figure 1A. Subjects first learn this offset mapping using stimuli given in random order. Then, a 12-element repeating sequence is introduced without the subject's knowledge. The sequence is sufficiently long that subjects usually do not develop awareness that a sequential structure is present in the stimuli. However, they demonstrate significant improvement of response latency consistent with implicit learning. Next, the subjects are transferred to a standard compatible version of the SRT task in which there is a direct spatial correspondence of each cue and digit movement (first stimulus cues first finger movement and so on) as shown in Figure 1B. At transfer subjects are given stimuli in random order, then the just learned sequence is reintroduced in either of two forms. In the perceptual condition, the administered sequence is identical to that used during training. In this case, perceptual events are occur- 


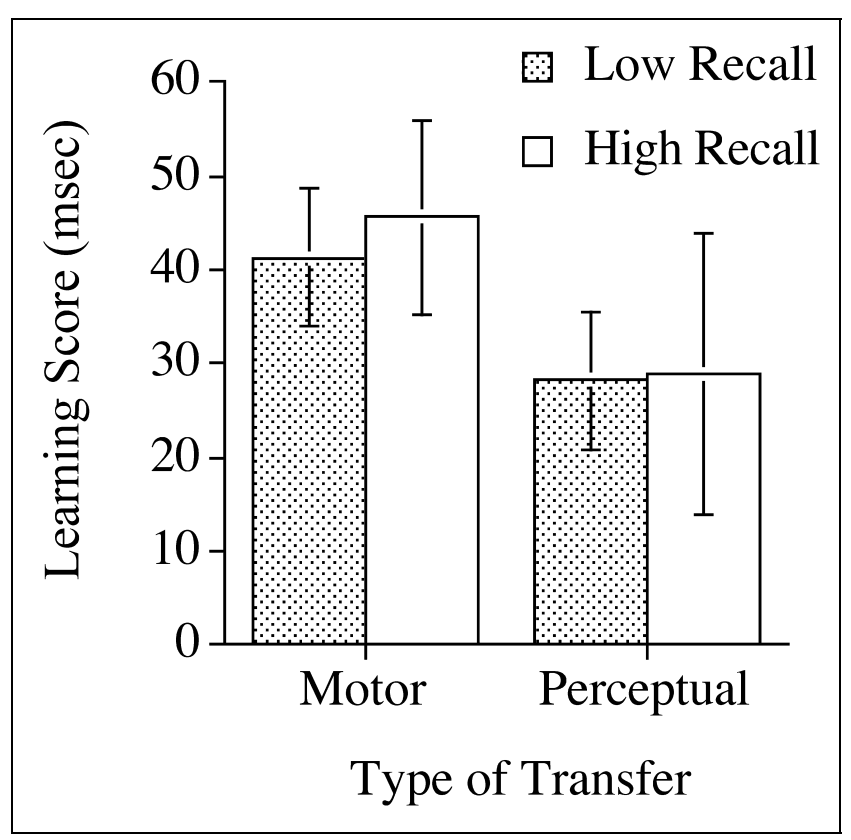

Figure 2. Changes of reaction time for the motor and perceptual transfer groups and by recall ability. Learning scores were created by subtracting the incompatible sequence (\#12) from the incompatible random block (\#13) at the end of the experiment. An ANOVA with type of transfer and recall revealed no significant differences in the learning scores of the motor and perceptual groups regardless of recall ability.

ring in the same order as previous but the order of finger responses is different. In the motor condition, a modified sequence is used. In this case, the perceptual sequence is adjusted so that finger responses are in identical order to what was learned previously. In behavioral studies, only the motor group demonstrates significant evidence for improved response latencies compared to a random sequence, suggesting that in this variant of the SRT task the subjects are learning the specific order of responses (Willingham, 1999; Willingham et al., 2000). This finding was the basis for the following imaging experiment in which the dissociation provided a method for identifying changes of brain activity that were specific to retrieval of a sequence learned at the motor response level. We hypothesized that response-based sequence retrieval would occur within areas previously implicated in sequencing at the perceptual and/or response-based level. Three areas have been consistently involved in sequence learning tasks: the supplementary motor area (SMA) and adjacent pre-SMA on the mesial wall of the frontal cortex, the dorsal premotor cortex on the lateral frontal cortex, and the inferior parietal lobule, particularly on the left side (Nakahara, Doya, \& Hikosaka, 2001; Sakai et al., 1998, 1999; Hazeltine, Grafton, \& Ivry, 1997; Grafton, Hazeltine, \& Ivry, 1995; Jenkins, Brooks, Nixon, Frackowiak, \& Passingham, 1994; Tanji, 1994, 1996). Given this knowledge, we could make a strong a priori prediction on the probable anatomic loci involved in this process.
Two other factors were also controlled for in the following imaging study. In previous work, we identified different patterns of activation for sequences learned under single- and dual-task conditions. This work demonstrated that both attentional distraction and the awareness of sequence structure interact with the neural substrates of sequence acquisition (Grafton et al., 1995; Grafton, Salidis, \& Willingham, 2001; Hazeltine et al., 1997). To minimize the impact of these complex interactions for interpreting functional imaging data, the task in the present study was performed without secondary distraction of attention. To minimize the potential confound of explicit awareness of the sequence on the underlying functional neuroanatomy, post hoc testing was used to differentiate subjects into high and low explicit recall of the specific sequence used during training. Subsequently, the image analysis focuses on those subjects demonstrating evidence for low recall.

\section{RESULTS}

\section{Behavioral Results}

We wanted to ensure that explicit knowledge of the stimulus sequence did not contaminate our results. We expected that subjects with explicit knowledge of the stimulus sequence would fail to demonstrate motor transfer, as during motor transfer, the stimulus sequence is different from that seen during training. However, subjects with explicit knowledge of the stim-

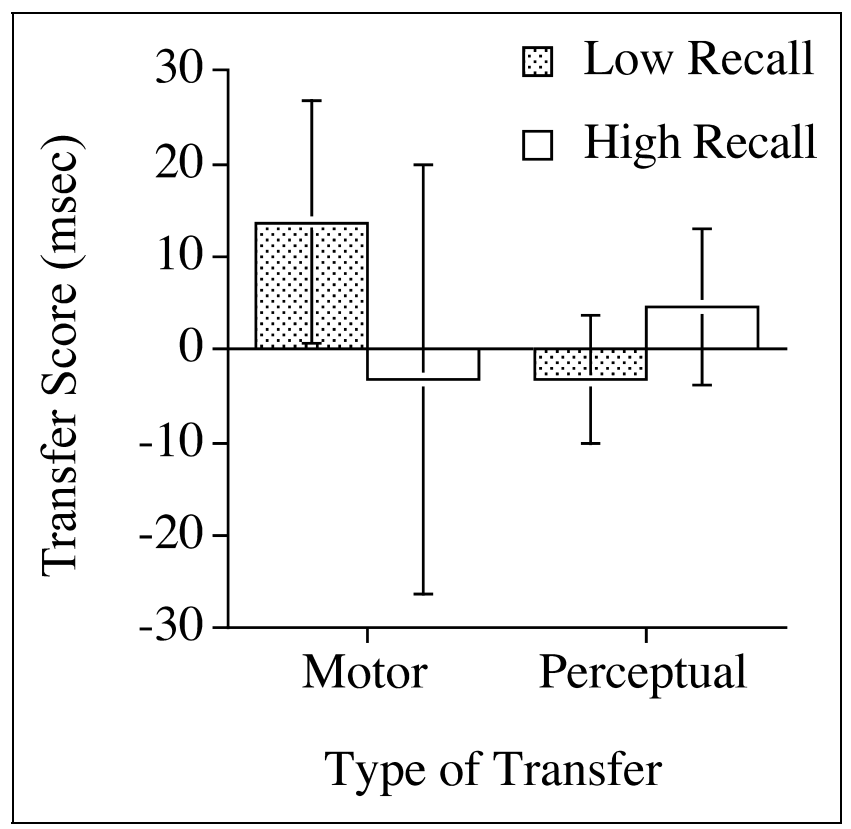

Figure 3. Interaction of transfer group (motor, perceptual) and level of recall on magnitude of transfer score (indicator of sequence retrieval after transfer to a spatially compatible mapping). Recall was determined as high or low based upon subject recall ability compared to a median score. No significant effects were evident $(F \mathrm{~S}<1)$ on a $2 \times 2$ ANOVA. 
ulus sequence should demonstrate perceptual transfer, as during perceptual transfer, the stimulus sequence is the same as that seen during training. We used subjects' free recall scores as a measure of explicit knowledge. Each subject's free recall score reflected the number of positions of the 12-unit stimulus sequence correctly recalled. A position was considered correct when it was included within a correctly recalled segment consisting of a minimum of three consecutive positions, but these recalled segments themselves need not be consecutive. For example, if a subject saw stimuli corresponding to positions 314324123142 and recalled 123143, the score would be 6 , because both 123 and 143 occurred in the sequence. Likewise, in this same example, if a subject recalled 4231, the subject would receive a recall score of 4 , as the sequence 4231 occurs once the presented sequence is appended to itself; the "beginning" of the sequence is arbitrary.

To aid in evaluating the recall scores, we assessed the effects of guessing on the recall task by rescoring each subject's free recall responses against a randomly selected sequence. As such, we were able to generate a measure of guessing performance. Mean number of positions correctly recalled on this measure of guessing performance was $4.7(S D=1.9)$, similar to the level of guessing performance found in other studies using the SRT task (e.g., Willingham \& Goedert-Eschmann, 1999). Overall, the motor group had marginally higher recall than the perceptual group $(M=6.17, S E=0.53$ for motor and $M=3.83, S E=0.99$ for perceptual), $t=2.06$, $p=.05$. To account for explicit knowledge of the sequence, subjects were divided into high- and low-recall groups based on their recall scores. Subjects with a recall score of 6 (the median score across all 24 subjects and less than $1 \mathrm{SD}$ above mean guessing performance) or lower were placed in the low-recall group $\left(N_{\text {LOW-MOTOR }}=\right.$ $\left.7, N_{\text {LOW-PERCEPTUAL }}=9\right)$ and those with a recall score of

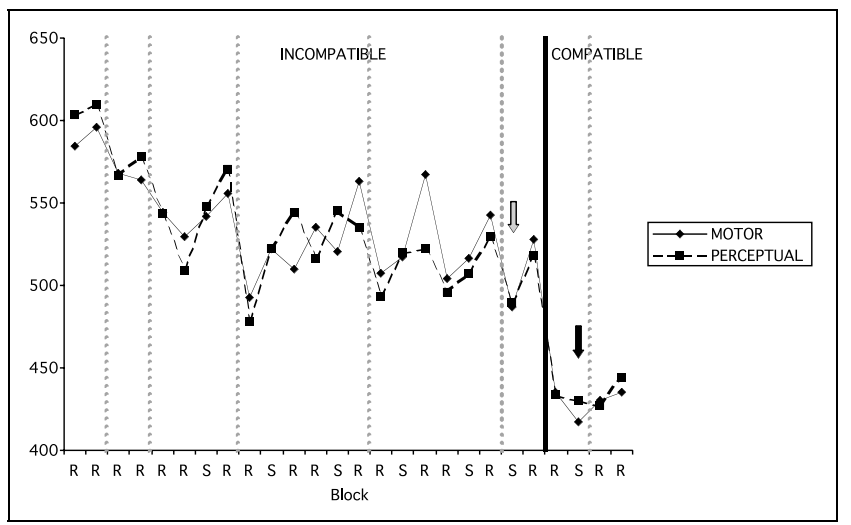

Figure 4. Behavioral results for the low-recall subjects categorized by transfer group (motor, perceptual). Only the motor group showed behavioral benefits at transfer. Paired sample $t$ tests suggested the difference between groups approached significance. Gray arrow indicates final incompatible sequence block; solid arrow indicates compatible sequence block.

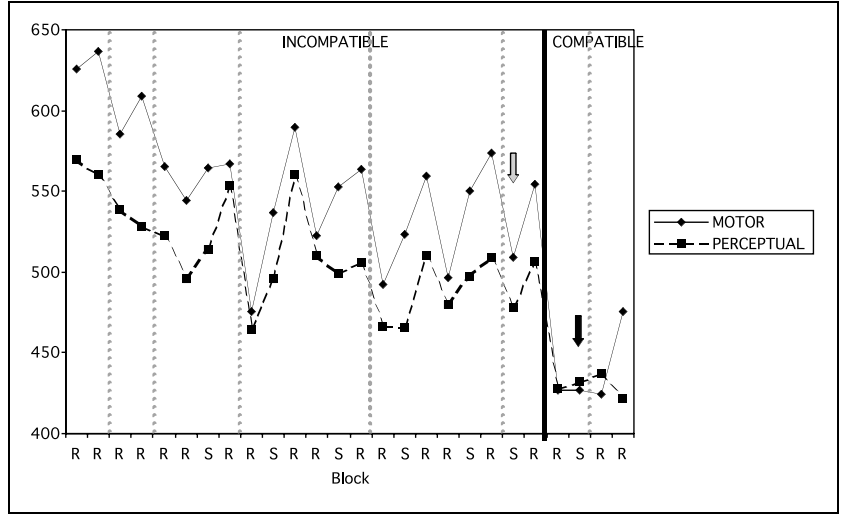

Figure 5. Behavioral performance for high-recall subjects categorized by transfer group (motor, perceptual). None of the subjects showed a behavioral learning benefit for the compatible sequence block, and there was no significant difference between the two groups. Gray arrow indicates final incompatible sequence block; solid arrow indicates compatible sequence block.

greater than 6 were placed in the high recall group $\left(N_{\text {HIGH-MOTOR }}=5, N_{\text {HIGH-PERCEPTUAL }}=3\right)$.

\section{Sequence Acquisition}

Learning scores were determined by subtracting reaction time (RT) performance during Block 12 from that in Block 13 (Figure 2). Overall, the learning scores were greater than zero $(M=35.8, S E=4.5, t=7.96, p<$ .001). An ANOVA on the learning scores with type of transfer (motor, perceptual) and recall (high, low) as factors revealed that there was no significant difference in the learning scores of the motor transfer and perceptual transfer groups regardless of recall ability, $F_{S}<2.3$, ps $>.15$.

\section{Sequence Retrieval after Transfer}

Transfer scores were determined by subtracting RT performance during Block 15 from that in Block 16 (Figure 3). A $2 \times 2$ ANOVA on the transfer scores with type of transfer (motor or perceptual) and recall (high or low) as factors yielded no significant effects, $F$ s $<1$. However, separate analysis of the transfer scores within each recall group revealed a marginal effect of type of transfer. For those subjects with low recall, only the motor group showed learning benefits at transfer as illustrated by improved RTs (Figure 4). The high-recall subjects, however, showed no learning benefit regardless of transfer type (Figure 5). Paired sample $t$ tests of RTs comparing the first compatible random block to the first compatible sequence block indicated that only the low-recall subjects demonstrated a marginally significant benefit for the sequence [low motor, $t(6)=1.67, p=.07$; low perceptual, $t(8)=0.35, p=.37$; high motor, $t(4)=$ $-0.02, p=.5$; high perceptual, $t(2)=-.32, p=.39$ ]. 
Given these behavioral findings, we focused upon the imaging results for the low-recall subjects.

\section{Functional Localization}

\section{Sequence Acquisition}

The low-recall subjects (regardless of subsequent group assignment in the motor or perceptual transfer group) demonstrated significant learning-related increases and decreases in neural structures previously associated with sequence learning. Using a random-effects statistical model, learning-related increases were observed in the left motor cortex and adjacent precentral sulcus (premotor cortex), bilateral medial aspect of the superior frontal gyrus (SMA) and adjacent cingulate motor area, bilateral middle frontal gyrus (dorsolateral prefrontal cortex $[\mathrm{DLPFC}])$, precuneus, and bilateral inferior parietal cortex at $p<.005$ (because of the a priori anatomic prediction, correction for comparisons across multiple locations was not performed) (Figure 6). Overall, these findings are similar to learning-related increases observed during acquisition of six-element sequences learned with a spatially compatible mapping with or without attentional distraction (Hazeltine et al., 1997; Grafton et al., 1995). Prominent decreases were seen in

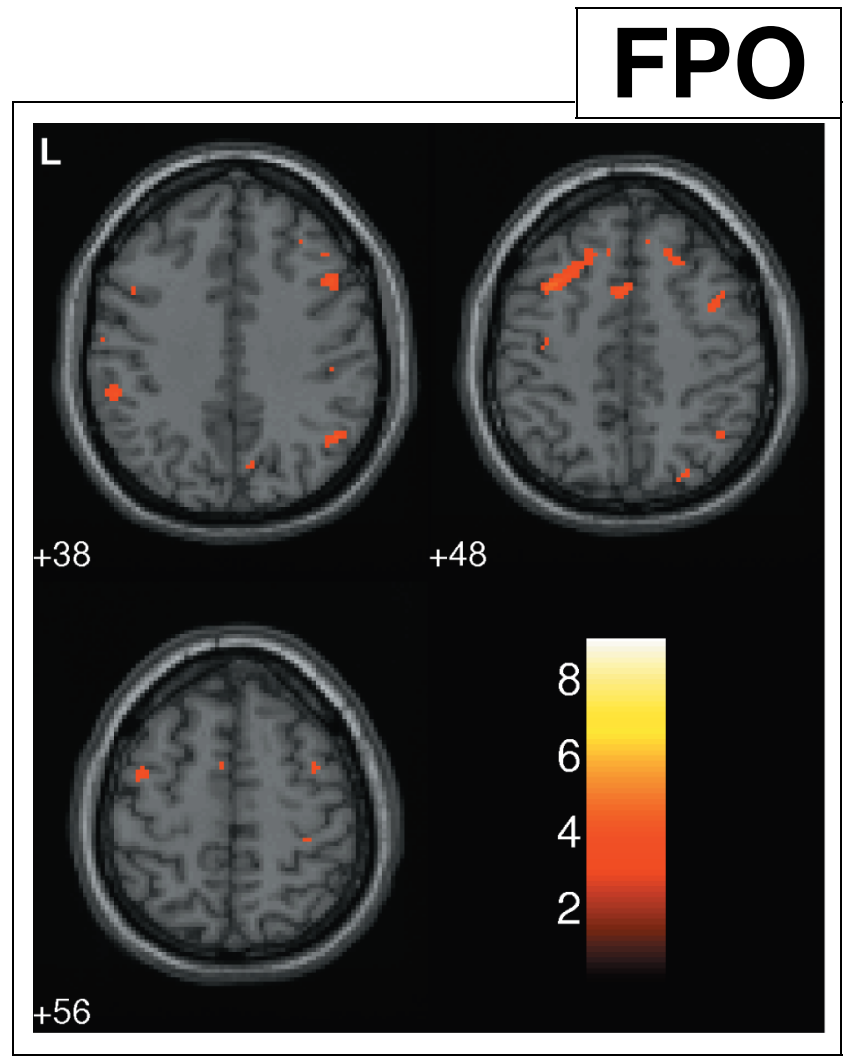

Figure 6. Learning-related increases of activity during sequence acquisition with a spatially incompatible mapping (Blocks 7-11). Results are for all low-recall subjects for $p<.005$ (uncorrected). Axial images demonstrate increasing activity over time in the bilateral inferior parietal lobule and precuneus $(z=38)$, in the bilateral medial and middle frontal gyri $(z=48)$, and in the left motor cortex $(z=56)$.

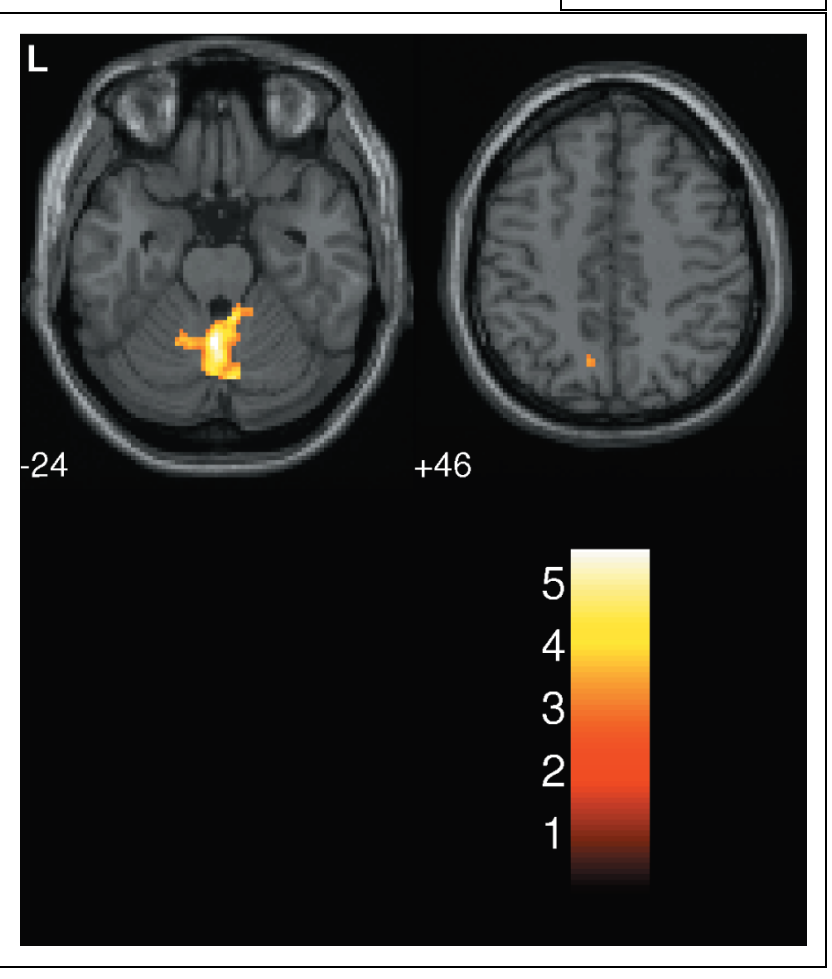

Figure 7. Learning-related decreases of activity during sequence acquisition with a spatially incompatible mapping (Blocks 7-11). Results are for all low-recall subjects. Axial images demonstrate decreasing activity over time in the bilateral anterior cerebellum $(z=-24)$ and in the left precuneus $(z=46)$ at $p<.005$

the anterior cerebellum and bilateral precuneus at $p<$ .005 (uncorrected) (Figure 7). The locations of all sites showing longitudinal changes of BOLD magnitude are summarized in Table 1. The learning-related contrasts shown here were weighted linearly; using a nonlinear RT-weighted contrast showed no major differences in size or location of learning-related changes and is therefore not presented here.

\section{Sequence Retrieval after Transfer}

The critical analysis compared the two transfer groups during retrieval of the previously learned sequence after transfer to a spatially compatible mapping. What brain areas are preferentially recruited in the motor group, that is, those demonstrating enhanced performance? Using a random-effects statistical model, we calculated an interaction of transfer group (motor or perceptual) by task (sequence vs. rest) and then applied a thresholding mask by each transfer group's significant main effect of compatible sequence block (\#15) > the 30-sec rest immediately preceding it. The mask is used to confirm that the appropriate transfer group sequence versus rest effect was driving the effect seen in the interaction. Again, this analysis was completed only for the low-recall subjects. 
Table 1. Localization of Longitudinal Increases and Decreases BOLD Magnitude during Sequence Acquisition Relative to Baseline across Training Blocks 7-11

\begin{tabular}{|c|c|c|c|c|c|}
\hline \multirow{2}{*}{$\frac{\text { Region }}{\text { Learning-related increases }}$} & \multirow[t]{2}{*}{ Cluster (voxels) } & \multirow[t]{2}{*}{ Z Score } & \multicolumn{3}{|c|}{ MNI Coordinates $\{x, y, z\}(\mathrm{mm})$} \\
\hline & & & & & \\
\hline Right medial aspect of superior frontal gyrus $(9 / 10)$ & 28 & 4.32 & 6 & 56 & 26 \\
\hline \multirow[t]{2}{*}{ Left superior/middle frontal gyrus (8/9) } & 112 & 4.62 & -34 & 14 & 48 \\
\hline & & 3.44 & -14 & 28 & 52 \\
\hline Bilateral medial aspect of superior frontal gyrus (6) & 20 & 4.06 & 0 & 12 & 48 \\
\hline \multirow[t]{2}{*}{ Bilateral medial aspect of superior frontal gyrus (6) } & 27 & 4.05 & 2 & 36 & 42 \\
\hline & & 3.25 & -4 & 42 & 40 \\
\hline Bilateral medial aspect of superior frontal gyrus (10) & 14 & 3.44 & 8 & 64 & 8 \\
\hline \multirow[t]{3}{*}{ Right middle frontal gyrus (8) } & 116 & 5.06 & 26 & 26 & 44 \\
\hline & & 3.85 & 22 & 26 & 52 \\
\hline & & 3.10 & 28 & 16 & 52 \\
\hline Right middle frontal gyrus (9) & 16 & 3.41 & 30 & 28 & 34 \\
\hline \multirow[t]{3}{*}{ Right middle frontal gyrus $(9 / 46)$} & 597 & 9.69 & 46 & 18 & 32 \\
\hline & & 6.89 & 48 & 28 & 20 \\
\hline & & 4.91 & 40 & 12 & 28 \\
\hline Right cingulate gyrus (23/31) & 14 & 3.32 & 4 & -48 & 24 \\
\hline Right precentral gyrus (4) & 28 & 3.82 & 30 & -28 & 62 \\
\hline Right precentral gyrus (6) & 20 & 3.77 & 48 & -14 & 30 \\
\hline Right paracentral lobule (6) & 10 & 3.60 & 8 & -32 & 70 \\
\hline Left precentral/middle frontal gyrus (6) & 23 & 3.72 & -40 & 0 & 56 \\
\hline Left precentral gyrus ( 4 ) & 22 & 3.82 & -62 & -12 & 26 \\
\hline Left postcentral gyrus (5) & 60 & 4.68 & -14 & -44 & 66 \\
\hline \multirow[t]{2}{*}{ Right superior/middle temporal gyrus (21/22) } & 150 & 5.10 & 50 & -20 & 4 \\
\hline & & 4.36 & 58 & -14 & 2 \\
\hline \multirow[t]{3}{*}{ Left superior/middle temporal gyrus (21/22) } & 132 & 5.85 & -58 & -22 & -2 \\
\hline & & 4.32 & -62 & -30 & -8 \\
\hline & & 3.49 & -64 & -14 & -2 \\
\hline Left superior/middle temporal gyrus (21/39) & 56 & 4.48 & -48 & -52 & 8 \\
\hline Left middle temporal gyrus (39) & 18 & 4.53 & -48 & -68 & 24 \\
\hline Right inferior parietal lobule (40) & 77 & 4.11 & 54 & -58 & 32 \\
\hline Left inferior parietal lobule (40) & 28 & 3.81 & -52 & -38 & 38 \\
\hline Bilateral precuneus (7) & 19 & 3.54 & 14 & -72 & 40 \\
\hline Left superior occipital gyrus (19) & 19 & 3.62 & -34 & -82 & 24 \\
\hline Right middle occipital gyrus (19) & 12 & 3.55 & 28 & -86 & 18 \\
\hline \multirow[t]{2}{*}{ Left middle occipital gyrus (18) } & 32 & 4.15 & -30 & -82 & 10 \\
\hline & & 3.55 & -26 & -86 & 4 \\
\hline \multirow[t]{2}{*}{ Left middle occipital/temporal gyrus (19/37) } & 12 & 3.65 & -42 & -68 & -14 \\
\hline & & 3.40 & -50 & -66 & -14 \\
\hline
\end{tabular}


Table 1. (continued)

\begin{tabular}{|c|c|c|c|c|c|}
\hline Region & Cluster (voxels) & Z Score & \multicolumn{3}{|c|}{ MNI Coordinates $\{x, y, z\}(\mathrm{mm})$} \\
\hline Right lingual gyrus (18) & 10 & 3.50 & 20 & -76 & -12 \\
\hline Right lingual gyrus (18) & 10 & 3.50 & 20 & -76 & -12 \\
\hline Left lingual/fusiform gyrus (18/19) & 15 & 3.88 & -24 & -70 & \\
\hline
\end{tabular}

\section{Learning-related decreases}

Right inferior frontal gyrus (47)

Right posterior cingulate/cuneus (30)

Right superior occipital gyrus (18)

Left posterior cingulate (30)

Right superior temporal gyrus (38)

Left superior temporal gyrus (38)

$\begin{array}{rrrrr}41 & 3.98 & 26 & 16 & -16 \\ 91 & 4.59 & 22 & -66 & 8 \\ & 4.14 & 24 & -76 & 16 \\ 15 & 3.74 & -24 & -70 & 10 \\ 18 & 3.90 & 44 & 12 & -18 \\ 19 & 3.73 & -44 & 6 & -22 \\ & 3.07 & 48 & 6 & -16 \\ 11 & 3.76 & -10 & -66 & 46 \\ 17 & 3.39 & -16 & -64 & -16 \\ 18 & 3.99 & 34 & 10 & -4 \\ 13 & 3.56 & 0 & -54 & -24 \\ 746 & 5.90 & 2 & -62 & -22 \\ & 5.33 & 8 & -44 & -24 \\ & 4.89 & 36 & -66 & -36 \\ 10 & 3.41 & & & -36 \\ \end{array}$

Right middle temporal gyrus (37)

Left precuneus (7)

Left parahippocampal gyrus (37)

Right insula

Bilateral lobule V/VI/IX

Right lobule VI

Results are for all low-recall subjects. Co-ordinates are from the MNI template and use the same orientation and origin as found in the Talairach and Tournoux (1988) atlas. The table shows at most local maxima $>8.00 \mathrm{~mm}$ apart per cluster $(k \geq 10)$ at $p<.005$ (uncorrected). Brodmann's area notation is indicated in parentheses.

Areas that are more active during successful sequence retrieval in the motor transfer group relative to the perceptual transfer group at $p<.005$ (uncorrected) are shown in Figure 8. Retrieval of sequential responses is associated with activity in the bilateral SMA and underlying cingulate cortex, bilateral inferior frontal gyrus (ventral PMC), left caudate and left inferior parietal lobule, summarized in Table 2. The perceptual transfer group, with whom there was no performance improvement indicative of successful sequence retrieval, failed to achieve significant activation at $p<.005$ or at $p<.01$ (uncorrected).

\section{DISCUSSION}

The central finding of this experiment is that the implicit retrieval of sequential information that is specific for a series of actions rather than perceptual events is associated with activation of the SMA, the inferior parietal cortex, and the premotor cortex. This finding provides specificity to previous human functional imaging studies of sequence learning by establishing that recruitment of these systems is linked to the temporal order of actions and not to more general sensory to motor transformations or the temporal order of instructions. The findings, observed in those subjects with low recall for sequence structure, extend previous imaging studies that demonstrated recruitment of the SMA and the premotor cortex by establishing that this response-based learning can occur under implicit conditions. The results do not exclude the possibility that these areas may also be involved in explicit sequence learning and trial and error learning as well. While ostensibly there is the possibility that subjects in our low-motor group had explicit knowledge of the motor sequence rather than the response sequence, as our free-recall test assessed knowledge only of the stimulus locations, similar patterns of transfer in the SRT task are witnessed in other behavioral studies when there is little knowledge of either the perceptual or motor sequence (Willingham, 1999).

The behavioral results in the low-recall group further support the notion that sequence learning can be response-based rather than perceptual-based. Response-based learning may be further categorized 


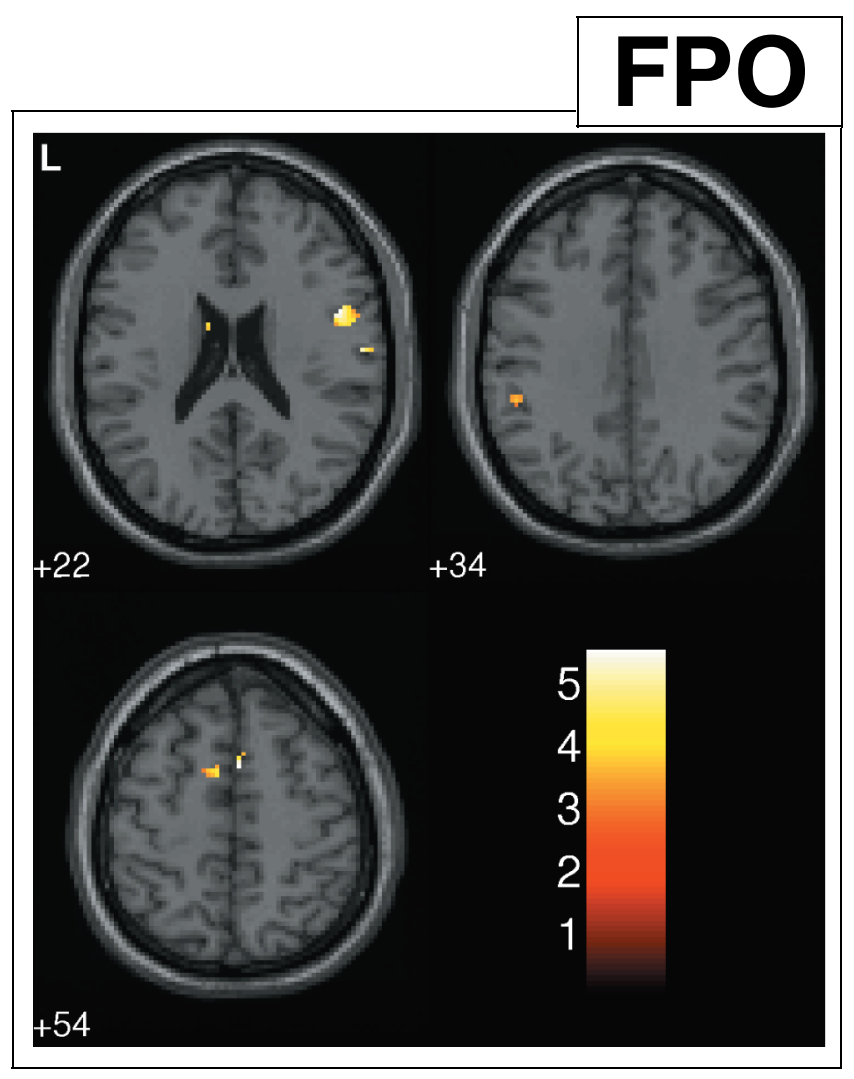

Figure 8. Functional localization of successful motor-based sequence retrieval. Axial images demonstrate regions with greater activity during performance of a sequence block (\#15) versus the preceding 30-sec rest block for the motor transfer group relative to the perceptual transfer group. Results are for all motor low-recall subjects after transfer to a spatially compatible mapping for $p<.005$ (uncorrected) Areas of activation included the premotor cortex $(z=22)$, the left inferior parietal lobule $(z=34)$, and the bilateral SMA $(z=54)$.

behaviorally into goal- and movement-based learning. In goal-based sequence learning, the performance improvements are linked to the outcome of an action (Hazeltine, 2002; Cohen et al., 1990). In movementbased sequence learning, they are linked to specific effectors or actions (Willingham, 1999; Willingham et al., 2000). For the SRT task, the action (finger movement) and its outcome (a key press) are indistin- guishable so we cannot make a distinction between these two types of response-based learning. Analogous distinctions between movement- and goal-based learning have been difficult to draw from existing neurophysiological experiments in nonhuman primates, where the sequence of movements and their outcomes are closely coupled. However, in previous variants of the SRT task, there is strong evidence for transfer across effectors, suggesting that under typical training conditions, subjects are not learning at a movement-based level of representation (Grafton, Hazeltine, \& Ivry, 1998; Grafton et al., 2001; Cohen et al., 1990). This leaves open the interpretation that SRT learning is primarily goal-based, that is, subjects learn sequences of general actions that are suitable for achieving particular goals.

Recent neuroimaging and neuronal recording studies have implicated the pre-SMA (anterior middle frontal gyrus) in the acquisition of visuomotor transformations (Sakai et al., 1999; Nakamura, Sakai, \& Hikosaka, 1998) and the acquisition and retrieval of action sequences in SMA-proper (Honda et al., 1998; Nakamura et al., 1998; Tanji \& Shima, 1994; Jenkins et al., 1994). Our results are consistent with this. We saw SMA-proper activation within the low-recall motor transfer group when examining the compatible sequence versus rest conditions; this was not the case for the low-recall perceptual transfer group. The motor transfer group also activated the bilateral posterior inferior frontal gyrus (i.e., ventral premotor cortex). This site is also activated during trial and error learning, conditional learning, and movement tasks involving the distal arm and hand (Grafton et al., 1995, 1998; Hazeltine et al., 1997; Rauch et al., 1997; Jenkins et al., 1994; Mitz, Godschalk, \& Wise, 1991). It is also active during the imitation of explicit goal-oriented actions in both humans (Koski et al., 2002) and monkey (Rizzolatti, Fadiga, Gallese, \& Fogassi, 1996). This activation is particularly strong for contralateral movements to those observed (Koski et al., 2002). Our experiment requires the subject to retrieve a goal-based motor response but requires the subject to transform the

Table 2. Localization of BOLD Magnitude Increases during Sequence Retrieval that are Greater for Subjects in the Motor Transfer Group Relative to the Perceptual Transfer Group after Transfer to a Spatially Compatible Mapping (Block 15)

\begin{tabular}{lccrc}
\hline Region & Clusters (Voxels) & Z Score & \multicolumn{2}{c}{ MNI Coordinates $\{x, y, z\}($ mm) } \\
\hline Right medial aspect of superior frontal gyrus (6/8) & 27 & 5.58 & 4 & 8 \\
Left medial aspect of superior frontal gyrus (6) & 13 & 4.35 & -6 & 6 \\
Right inferior frontal gyrus (9/44) & 93 & 5.47 & 50 & 6 \\
Left cingulate gyrus (32) & 12 & 3.90 & -10 & -50 \\
Left inferior parietal lobule (40) & 22 & 3.65 & -10 & -36 \\
Left caudate & 36 & 4.21 & 34 \\
\hline
\end{tabular}

Results are for low-recall subjects only. Co-ordinates are from the MNI template and use the same orientation and origin as found in the Talairach and Tournoux (1988) atlas. The table shows at most local maxima $>8.00 \mathrm{~mm}$ apart per cluster $(k \geq 10)$ for $p<.005$ (uncorrected). Brodmann's area notation is indicated in parentheses. 
observed goal from the incompatible mapping to the compatible mapping. This supports the more general concept that the ventral premotor cortex is responsible for transformation from extrinsic to intrinsic co-ordinates in goal-based actions. Finally, activation of the inferior parietal lobule was observed with sequence retrieval in the motor transfer group. This area is associated with action representation, and lesions to this area lead to apraxia as well as errors in the sequencing of goal-specific actions (Haaland, Harrington, \& Knight, 1999). Together these anatomically and functionally interconnected areas form a distributed circuit for storing and retrieving sequences of goal-oriented actions. In contrast to the motor transfer group, the perceptual transfer group, in whom there was no behavioral evidence for successful sequence retrieval after transfer, failed to show significant activation. This provides further support that sequence retrieval is goal-related rather than perceptually oriented.

The experiment also tested for progressive changes of neural activity as subjects acquired a sequence that was learned using an incompatible sensory to motor mapping. The results demonstrate overlap with previous studies of sequence learning with a spatially compatible sensory to motor mapping. In particular, increasing activity in motor-related areas including the motor cortex, the SMA, and the inferior parietal cortex was observed during learning under conditions of attentional distraction in which a secondary counting task was performed (Grafton et al., 1995, 1998; Hazeltine et al., 1997). Involvement of working memory and more cognitive representational systems in the prefrontal cortex and temporal areas was observed under conditions without these secondary tasks (Hazeltine et al., 1997; Grafton et al., 1995). The current results demonstrate that all of these neural systems can become engaged during sequence acquisition when a distractor is not present.

During incompatible learning, subjects not only learn the sequence implicitly but are also learning a nonstandard stimulus-response mapping. Our incompatible sequence learning results for the low-recall subjects also identified learning-related increases within the inferior, middle, and medial frontal gyri extending into the dorsal premotor cortex, and the anterior cingulate, with learning-related decreases within the cerebellum and the precuneus. Both the posterior frontal gyrus (dorsal PMC) and the anterior cerebellum have been implicated in stimulus-response mapping. The dorsal PMC has been shown to underlie associative motor learning in both nonhuman primates (Wise, 1996; Mitz et al., 1991) and in neuroimaging studies of humans (Toni \& Passingham, 1999; Grafton et al., 1998; Deiber et al., 1997; Hazeltine et al., 1997). The cerebellum has long been associated with sensorimotor co-ordination, adaptation, and associative learning (Thach, 1997; Ito, 1984; Brooks \& Thach, 1981; Albus, 1971). Both anatomical (Middleton \& Strick, 1997, 2000, 2001; Schmahmann \& Pandya, 1997) and functional (Bischoff-Grethe, Ivry, \& Grafton,
2002) evidence support its role in stimulus-response mapping. In our experiment, the associated changes within the PMC and the cerebellum are likely due to learning the stimulus-response mapping in parallel with sequence learning.

Our incompatible learning experiment supports previously recorded behavioral evidence suggesting that implicit learning is response based rather than perceptual based. Subjects indicated a performance benefit upon transfer to the compatible mapping only when the sequence was consistent in the response domain across mappings. Functionally, both groups indicated activation in areas commonly associated with implicit sequence learning. Posttransfer, subjects in the motor group activated the SMA and cingulate areas commonly linked to the retrieval of motor sequences, whereas the perceptual group failed to show significant activation. The experiment lends support to the model described by Nakahara et al. (2001), but in particular, it provides specificity to sequencing performance by indicating that motor retrieval is related to sequential responses rather than sensory or temporal information.

\section{METHODS \\ Experimental Design}

Twenty-four right-handed participants (9 men, 15 women) aged 18 to 39 (average age $=19.8$ years) gave written informed consent. Subjects rested the four fingers of their right hand on the four keys of the response board. Four squares, arranged horizontally, appeared on the projection screen. Subjects responded when a square filled white by pressing a key on the response board that corresponded to that stimulus location. One stimulus appeared every $1000 \mathrm{msec}$ and remained on the projection screen for $1000 \mathrm{msec}$. Stimulus locations were mapped to response locations in one of two ways. During sequence learning, all subjects responded with an incompatible mapping between the stimulus and the response; subjects responded by pressing the key one position to the right of the stimulus. If the stimulus appeared in the rightmost position, subjects "wrapped around" and pressed the key on the far left (Figure 1A). At transfer, subjects responded with a compatible mapping by pressing the key that directly corresponded to the stimulus position (Figure 1B). For the perceptual group, the stimulus sequence was maintained at transfer; they pushed a different key sequence, but saw the same visuospatial sequence as during training. For the motor group, the stimulus sequence was changed at transfer; they pushed the same key sequence, but saw a different visuospatial sequence. Subjects were not informed of the repeating sequence in the SRT, but were told to respond on each trial as quickly and as accurately as possible. 
For each subject, a sequence was selected randomly from a corpus of 563 twelve-unit sequences that met the following criteria: a stimulus could not repeat itself (e.g., 1332); each stimulus appeared an equal number of times in the sequence; and the sequence could not contain runs of four units (e.g., 1234) or trills of four units (e.g., 2424). Each block of SRT consisted of 96 trials. Random blocks were created pseudorandomly with the stipulation that the entire random block met the same criteria as detailed above.

All subjects performed 17 blocks of SRT (Table 3) interspersed with 30-sec rest blocks, during which the subject was instructed to make no responses. Rest blocks were indicated to the subject by a large "X" across the stimuli on the screen. The first block of the SRT was random and was performed outside the scanner to give subjects practice with the incompatible mapping. The remaining blocks were performed in the scanner. Blocks 2 through 6 consisted of randomly appearing stimuli and again were intended to give subjects practice at the incompatible mapping prior to introducing the sequence. Blocks 7 through 11 were sequence training blocks in which the subjects responded to three full repetitions of the sequence embedded between short blocks of random trials. The embedding of the sequenced trials within random trials was intended to reduce explicit knowledge of the sequence. Behavioral evidence of sequence learning with an incompatible mapping was assessed by comparing RTs during Block 12, an all-sequenced block, and Block 13, an all-random block. In Blocks 14 (all random), 15 (all sequenced), and Blocks 16 and 17 (both all random) subjects responded with a compatible mapping. Evidence for successful retrieval of the previously learned sequence after transfer to the compatible mapping was assessed by comparing RT during Blocks 15 (sequence) and 16 (random).

To assess whether subjects became explicitly aware of the sequence during the SRT training, all subjects were told during debriefing that they may or may not have been in a group that saw a repeating sequence of stimuli during initial training. They were asked to guess as to which group they belonged. Most subjects $(N=21)$ guessed they were with the pattern group. Subsequently, a free recall task was administered to assess explicit knowledge of the sequence. Subjects were told that during training the stimuli had sometimes appeared in a 12-unit repeating sequence and were asked to recall as much of the sequence of stimulus locations as possible. Subjects were given a sheet of paper on which there were 12 rows consisting of four columns, with each row corresponding to a unit of the repeating sequence and each column corresponding to one of the four spatial locations on the screen. Subjects indicated where a stimulus had appeared by putting an $X$ in the corresponding column.

\section{Magnetic Resonance Imaging}

Experiments were performed on a General Electric Horizon whole-body "Echospeed" 1.5-T MRI scanner (Kwong et al., 1992; Ogawa et al., 1992). A tightly fitted foam pillow was used to restrict head motion. After a nine-plane orthogonal localizer series is acquired (9 sec), a low-resolution T1-weighted axial fast spin-echo study (TE Min full, TR $650 \mathrm{msec}$, echo train 2, FOV $24 \mathrm{~cm}$, $5.5 \mathrm{~mm}$ slice with $0 \mathrm{~mm}$ gap, 27 contiguous slices) that is coplanar to subsequent functional runs was acquired (2 min). This is used for between-session coregistration and characterization of BOLD-EPI image distortion. Functional MRI was performed with gradient-recalled echo-planar imaging: TE $35 \mathrm{msec}$, TR $2000 \mathrm{msec}$, flip angle $90^{\circ}$, phase/frequency encoding $64 \times 64$, FOV $24 \mathrm{~cm}$ with ramp sampling. Eight fMRI sessions of 112 scans each were obtained. High-resolution structural images were acquired with a modified inversion prepared fast gradient-echo sequence: $\mathrm{TI}=600 \mathrm{msec}$, TD $=$ $1200 \mathrm{msec}, \mathrm{TR}=7.6 \mathrm{msec}, \mathrm{TE}=3 \mathrm{msec}$. A 3-D volumetric scan was acquired with 124 sagittal slices, $1.2 \mathrm{~mm}$ thick, $256 \times 192$ matrix, FOV $=250 \mathrm{~mm}$.

\section{Statistical Analysis}

The data were analyzed using Statistical Parametric Mapping (SPM99, Wellcome Department of Cognitive Neurology, London, UK) (Friston et al., 1995). Motion-

Table 3. The Training Paradigm Used in the Experiment

\begin{tabular}{lllllllllllllllllll}
\hline Block & 1 & 2 & 3 & 4 & 5 & 6 & 7 & 8 & 9 & 10 & 11 & 12 & 13 & 14 & 15 & 16 & 17 \\
\hline Motor & $\mathrm{C} / \mathrm{I}$ & $\mathrm{I}$ & $\mathrm{I}$ & $\mathrm{I}$ & $\mathrm{I}$ & $\mathrm{I}$ & $\mathrm{I}$ & $\mathrm{I}$ & $\mathrm{I}$ & $\mathrm{I}$ & $\mathrm{I}$ & $\mathrm{I}$ & $\mathrm{I}$ & $\mathrm{C}_{\mathrm{m}}$ & $\mathrm{C}_{\mathrm{m}}$ & $\mathrm{C}_{\mathrm{m}}$ & $\mathrm{C}_{\mathrm{m}}$ \\
Perceptual & $\mathrm{C} / \mathrm{I}$ & $\mathrm{I}$ & $\mathrm{I}$ & $\mathrm{I}$ & $\mathrm{I}$ & $\mathrm{I}$ & $\mathrm{I}$ & $\mathrm{I}$ & $\mathrm{I}$ & $\mathrm{I}$ & $\mathrm{I}$ & $\mathrm{I}$ & $\mathrm{I}$ & $\mathrm{C}_{\mathrm{p}}$ & $\mathrm{C}_{\mathrm{p}}$ & $\mathrm{C}_{\mathrm{p}}$ & $\mathrm{C}_{\mathrm{p}}$ \\
Stimuli & $\mathrm{R}$ & $\mathrm{R}$ & $\mathrm{R}$ & $\mathrm{R}$ & $\mathrm{R}$ & $\mathrm{R}$ & $\mathrm{S}^{*}$ & $\mathrm{~S} *$ & $\mathrm{~S} *$ & $\mathrm{~S}^{*}$ & $\mathrm{~S} *$ & $\mathrm{~S}$ & $\mathrm{R}$ & $\mathrm{R}$ & $\mathrm{S}$ & $\mathrm{R}$ & $\mathrm{R}$ \\
Scan & 0 & 1 & 1 & 2 & 2 & 3 & 3 & 4 & 4 & 5 & 5 & 6 & 6 & 7 & 7 & 8 & 8 \\
\hline
\end{tabular}

Subjects performed a total of 17 task blocks, including one practice session. In the practice session, subjects performed 20 random trials of the compatible mapping followed by 96 random trials of the incompatible mapping within the scanner, but images were not acquired. The remaining blocks each consisted of 96 trials. Rest blocks (not shown) of 30 sec occurred between each behavioral block. $\mathrm{C}=$ compatible mapping; $\mathrm{C}_{\mathrm{m}}=$ compatible mapping in the motor domain; $\mathrm{C}_{\mathrm{p}}=$ compatible mapping in the perceptual domain; $\mathrm{I}=$ incompatible mapping; $\mathrm{R}=$ random trials: $\mathrm{S}=$ sequence trials; $\mathrm{S}^{*}=$ sequence trials composed of $12 \mathrm{R}-72 \mathrm{~S}-12 \mathrm{R}$ individual trials. 
correction to the first functional scan was performed within subject using a six-parameter rigid-body transformation. The mean of the motion-corrected images was coregistered to the individual's coplanar MRI using mutual information, followed by coregistration of the coplanar and high-resolution structural MRIs. The highresolution MRI (followed by the coregistered functional images) was then spatially normalized to the Montreal Neurologic Institute (MNI) template (Talairach \& Tournoux, 1988) by applying a 12-parameter affine transformation followed by a nonlinear warping using basis functions (Ashburner \& Friston, 1999). The spatially normalized functional scans were then smoothed with a 6-mm isotropic gaussian kernel to accommodate anatomical differences across participants.

\section{Experimental Design Matrix}

Subjects were evaluated under two categories:

(1) Level of recall. Subjects capable of recalling six or more elements of the 12-element sequence at the completion of the study were placed within the highrecall group; all others were placed within the low-recall group.

(2) Transfer group. After training with an incompatible mapping, subjects were split into two groups on transfer to the compatible mapping. One group was tested with an identical sequence of motor responses, the other with an identical sequence of perceptual events.

Two main effects were studied.

\section{Sequence Acquisition}

Longitudinal changes of brain activity during sequence learning with the incompatible mapping were evaluated using a set of linear contrasts across sequence Blocks 7 through 11. Both increases and decreases of BOLD magnitude over training were identified. The data were analyzed for all of the low-recall subjects (irrespective of subsequent placement in a motor or perceptual group) using random effects (Friston, Holmes, \& Worsley, 1999) at $p<.005$ (uncorrected). Because there were far fewer subjects in the high-recall group, detailed statistical analysis of this group is not included in this report.

\section{Sequence Retrieval after Transfer}

This was an interaction of transfer group (perceptual vs. motor) and retrieval of a sequence (i.e., differences between sequencing and rest after transfer to the compatible mapping). To further characterize the statistical map formed by the interaction term, the statistical images were further masked to show only those areas demonstrating significant main effects of task (sequence retrieval vs. rest) for each transfer group. Analysis was restricted to the low-recall group using random effects
(Friston et al., 1999) at $p<.005$ (uncorrected). Because there were far fewer subjects in the high-recall group, detailed statistical analysis of this group are not included in this report.

\section{Acknowledgments}

Supported by PHS grant NS33504.

Reprint requests should be sent to Scott T. Grafton, Center for Cognitive Neuroscience, 6162 Moore Hall, Dartmouth College, Hanover, NH 03755, or via e-mail: Scott.T.Grafton@dartmouth. edu.

The data reported in this experiment have been deposited in the fMRI Data Center (http://www.fmridc.org). The accession number is 2-2003-1141C.

\section{REFERENCES}

Albus, J. S. (1971). The theory of cerebellar function. Mathematical Biosciences, 10, 25-61.

Ashburner, J., \& Friston, K. J. (1999). Nonlinear spatial normalization using basis functions. Human Brain Mapping, 7, 254-266.

Bapi, R. S., Doya, K., \& Harner, A. M. (2000). Evidence for effector independent and dependent representations and their differential time course of acquisition during motor sequence learning. Experimental Brain Research, 132, 149-162.

Bischoff-Grethe, A., Ivry, R. B., \& Grafton, S. T. (2002). Cerebellar involvement in response reassignment rather than attention. Journal of Neuroscience, 22, 536-553.

Brooks, V. B., \& Thach, W. T. (1981). Cerebellar control of posture and movement. In V. B. Brooks (Ed.), Handbook of physiology, Section 1: The nervous system: Vol. 2. Motor control, part 2 (pp. 877-946). Bethesda, MA: American Physiological Society.

Cohen, A., Ivry, R. I., \& Keele, S. W. (1990). Attention and structure in sequence learning. Journal of Experimental Psychology: Learning, Memory, and Cognition, 16, 17-30.

Deiber, M. P., Wise, S. P., Honda, M., Catalan, M. J., Grafman, J., \& Hallett, M. (1997). Frontal and parietal networks for conditional motor learning: A positron emission tomography study. J Neurophysiol, 78, 977-991.

Friston, K. J., Holmes, A. P., \& Worsley, K. J. (1999). How many subjects constitute a study? Neuroimage, 10, 1-5.

Friston, K. J., Holmes, A. P., Worsley, K. J., Poline, J.-B., Frith, C. D., \& Frackowiak, R. S. J. (1995). Statistical parametric maps in functional imaging: A general linear approach. Human Brain Mapping, 2, 189-210.

Grafton, S. T., Hazeltine, E., \& Ivry, R. (1995). Functional mapping of sequence learning in normal humans. Journal of Cognitive Neuroscience, 7, 497-510.

Grafton, S. T., Hazeltine, E., \& Ivry, R. B. (1998). Abstract and effector-specific representations of motor sequences identified with PET. Journal of Neuroscience, 18, 9420-9428.

Grafton, S. T., Salidis, J., \& Willingham, D. B. (2001). Motor learning of compatible and incompatible visuomotor maps. Journal of Cognitive Neuroscience, 13, 217-231.

Haaland, K. Y., Harrington, D. L., \& Knight, R. T. (1999). Spatial deficits in ideomotor limb apraxia. A kinematic analysis of aiming movements. Brain, 122, 1169-1182.

Hazeltine, E. (2002). The representational nature of sequence learning: Evidence for goal-based codes. In B. Hommel 
(Ed.), Common mechanisms in perception and action: Attention and performance XIX (pp. 673-689). Oxford: Oxford University Press.

Hazeltine, E., Grafton, S. T., \& Ivry, R. (1997). Attention and stimulus characteristics determine the locus of motor-sequence encoding. A PET study. Brain, 120, 123-140.

Honda, M., Deiber, M. P., Ibanez, V., Pascual-Leone, A., Zhuang, P., \& Hallett, M. (1998). Dynamic cortical involvement in implicit and explicit motor sequence learning. A PET study. Brain, 121, 2159-2173.

Ito, M. (1984). The cerebellum and neural control. New York: Raven Press.

Jenkins, I. H., Brooks, D. J., Nixon, P. D., Frackowiak, R. S. J., \& Passingham, R. E. (1994). Motor sequence learning: A study with positron emission tomography. Journal of

Neuroscience, 14, 3775-3790.

Keele, S. W., Jennings, P., Jones, S., Caulton, D., \& Cohen, A. (1995). On the modularity of sequence representation. Journal of Motor Behavior, 27, 17-30.

Koch, I., \& Hoffmann, J. (2000). The role of stimulus-based and response-based spatial information in sequence learning. Journal of Experimental Psychology: Learning, Memory, and Cognition, 26, 863-882.

Koski, L., Wohlschlager, A., Bekkering, H., Woods, R. P., Dubeau, M. C., Mazziotta, J. C., \& Iacoboni, M. (2002). Modulation of motor and premotor activity during imitation of target-directed actions. Cerebral Cortex, 12, 847-855.

Kwong, K. K., Belliveau, J. W., Chesler, D. A., Goldberg, I. E., Weisskoff, R. M., Poncelet, B. P., Kennedy, D. N., Hoppel, B. E., Cohen, M. S., Turner, R., Cheng, H., Brady, T. J., Rosen, B R. (1992). Dynamic magnetic resonance imaging of human brain activity during primary sensory stimulation. Proceedings of the National Academy of Sciences, U.S.A., 89, 5675-5679.

Middleton, F. A., \& Strick, P. L. (1997). Dentate output channels: Motor and cognitive components. Progress in Brain Research, 114, 553-566.

Middleton, F. A., \& Strick, P. L. (2000). Basal ganglia and cerebellar loops: Motor and cognitive circuits. Brain Research, Brain Research Reviews, 31, 236-250.

Middleton, F. A., \& Strick, P. L. (2001). Cerebellar projections to the prefrontal cortex of the primate. Journal of Neuroscience, 21, 700-712.

Mitz, A. R., Godschalk, M., \& Wise, S. P. (1991). Learning-dependent neuronal activity in the premotor cortex: Activity during the acquisition of conditional motor associations. Journal of Neuroscience, 11, 1855-1872.

Nakahara, H., Doya, K., \& Hikosaka, O. (2001). Parallel cortico-basal ganglia mechanisms for acquisition and execution of visuomotor sequences-A computational approach. Journal of Cognitive Neuroscience, 13, 626-647.

Nakamura, K., Sakai, K., \& Hikosaka, O. (1998). Neuronal activity in medial frontal cortex during learning of sequential procedures. Journal of Neurophysiology, 80, 2671-2687.
Nattkemper, D., \& Prinz, W. (1997). Stimulus and response anticipation in a serial reaction time task. Psychological Research, 60, 98-112.

Ogawa, S., Tank, D. W., Menon, R., Ellermann, J. M., Kim, S. G., Merkle, H., Ugurbil, K. (1992). Intrinsic signal changes accompanying sensory stimulation: Functional brain mapping with magnetic resonance imaging. Proceedings of the National Academy of Sciences, U.S.A., 89, 5951-5955.

Rauch, S. L., Whalen, P. J., Savage, C. R., Curran, T., Kendrick, A., Brown, H. D., Bush, G., Breiter, H. C., Rosen, B. R. (1997). Striatal recruitment during an implicit sequence learning task as measured by functional magnetic resonance imaging. Human Brain Mapping, 5, 124-132.

Rizzolatti, G., Fadiga, L., Gallese, V., \& Fogassi, L. (1996). Premotor cortex and the recognition of motor actions. Brain Research: Cognitive Brain Research, 3, 131-141.

Sakai, K., Hikosaka, O., Miyauchi, S., Sasaki, Y., Fujimaki, N., \& Putz, B. (1999). Presupplementary motor area activation during sequence learning reflects visuo-motor association. Journal of Neuroscience, 19, RC1.

Sakai, K., Hikosaka, O., Miyauchi, S., Takino, R., Sasaki, Y., \& Pütz, B. (1998). Transition of brain activation from frontal to parietal areas in visuomotor sequence learning. Journal of Neuroscience, 18, 1827-1840.

Schmahmann, J. D., \& Pandya, D. N. (1997). The cerebrocerebellar system. International Review of Neurobiology, 41, 31-60.

Talairach, J., \& Tournoux, P. (1988). Co-planar stereotaxic atlas of the brain. New York: Thieme Medical Publishers.

Tanji, J. (1994). The supplementary motor area in the cerebral cortex. Neuroscience Research, 19, 251-268.

Tanji, J. (1996). New concepts of the supplementary motor area. Current Opinion in Neurobiology, 6, 782-787.

Tanji, J., \& Shima, K. (1994). Role for supplementary motor area cells in planning several movements ahead. Nature, 371, 413-416.

Thach, W. T. (1997). Context-response linkage. International Review of Neurobiology, 41, 599-611.

Toni, I., \& Passingham, R. E. (1999). Prefrontal-basal ganglia pathways are involved in the learning of arbitrary visuomotor associations: A PET study. Experimental Brain Research, 127, 19-32.

Willingham, D. B. (1999). Implicit motor sequence learning is not purely perceptual. Memory and Cognition, 27, 561-572.

Willingham, D. B., \& Goedert-Eschmann, K. (1999). The relation between implicit and explicit learning: Evidence for parallel development. Psychological Science, 10, 531-534.

Willingham, D. B., Wells, L. A., Farrell, J. M., \& Stemwedel, M. E. (2000). Implicit motor sequence learning is represented in response locations. Memory and Cognition, 28, 366-375.

Wise, S. P. (1996). Evolution of neuronal activity during conditional motor learning. In S. P. Wise (Ed.), The acquisition of motor behavior in vertebrates (pp. 261-286). Cambridge: MIT Press. 


\section{AUTHOR QUERIES}

No Query. 